\title{
Quantification and Gene Expression Analysis of Histone Deacetylases in Common Bean during Rust Fungal Inoculation
}

\author{
Kalpalatha Melmaiee, ${ }^{1}$ Venu (Kal) Kalavacharla, ${ }^{1,2}$ Adrianne Brown, ${ }^{1}$ Antonette Todd, \\ Yaqoob Thurston, ${ }^{1,3}$ and Sathya Elavarthi ${ }^{1}$ \\ ${ }^{1}$ College of Agriculture and Related Sciences, Delaware State University, Dover, DE 19901, USA \\ ${ }^{2}$ Center for Integrated Biological \& Environmental Research (CIBER), Delaware State University, Dover, DE 19901, USA \\ ${ }^{3}$ Department of Plant Science, South Dakota State University, Brookings, SD 57007, USA \\ Correspondence should be addressed to Venu (Kal) Kalavacharla; vkalavacharla@desu.edu
}

Received 20 June 2015; Accepted 27 October 2015

Academic Editor: Feng Chen

Copyright (C) 2015 Kalpalatha Melmaiee et al. This is an open access article distributed under the Creative Commons Attribution License, which permits unrestricted use, distribution, and reproduction in any medium, provided the original work is properly cited.

\begin{abstract}
Histone deacetylases (HDACs) play an important role in plant growth, development, and defense processes and are one of the primary causes of epigenetic modifications in a genome. There was only one study reported on epigenetic modifications of the important legume crop, common bean, and its interaction with the fungal rust pathogen Uromyces appendiculatus prior to this project. We measured the total active HDACs levels in leaf tissues and observed expression patterns for the selected HDAC genes at 0,12 , and 84 hours after inoculation in mock inoculated and inoculated plants. Colorimetric analysis showed that the total amount of HDACs present in the leaf tissue decreased at 12 hours in inoculated plants compared to mock inoculated control plants. Gene expression analyses indicated that the expression pattern of gene PvSRT1 is similar to the trend of total active HDACs in this time course experiment. Gene PvHDA6 showed increased expression in the inoculated plants during the time points measured. This is one of the first attempts to study expression levels of HDACs in economically important legumes in the context of plant pathogen interactions. Findings from our study will be helpful to understand trends of total active HDACs and expression patterns of these genes under study during biotic stress.
\end{abstract}

\section{Introduction}

Histone deacetylases are a family of enzymes that remove acetyl groups from lysine residues present in the N-terminal extension of core histones of nucleosomes [1] and have been found in bacteria, fungi, plants, and animals. Histone acetyltransferases (HATs) and deacetylases (HDACs) play an important role in chromatin structural modifications and epigenetic changes in many organisms. Research on histone deacetylase inhibitors (HDAC) began nearly 30 years ago when studies were laid out to understand why dimethyl sulfoxide (DMSO) caused terminal differentiation of murine erythroleukemia cells [2]. This early observation led to the development of novel pharmacological agents in the field of chromatin remodeling [1]. HDACs catalyze deacetylation reactions, which cause chromatin to coil by removing acetyl groups from lysine residues of histones. This deacetylation increases the positive charge on N-termini of the core histones. As a result, the interaction between core histones and negatively charged DNA increases which causes tight coiling of DNA, which in turn blocks access to the transcriptional machinery. The balance between the actions of HDACs, HATs, and transcriptional elements serves as a key regulatory mechanism for gene expression and in turn governs numerous developmental processes and disease states $[3,4]$.

HDACs are known to be involved in a myriad of plant physiological and developmental activities and in epigenetic events often for transcriptional repression of genes $[5,6]$. Several studies in plants have reported that there is a direct correlation of DNA methylation, histone deacetylation, and gene suppression $[5,7,8]$. In the model plant, Arabidopsis thaliana, HDA6 and MET1 interact directly to silence transposable elements by modifying DNA methylation, histone acetylation, and histone methylation status $[9,10]$. Genetic 
analysis in Arabidopsis indicates that HDA6 is a component of RdDM (RNA-directed DNA methylation) pathway [7]. Even in other systems like the African clawed frog Xenopus laevis, relaxation of methylated DNA in oocytes by the inhibition of histone deacetylases was observed $[8,11]$.

Removal of acetyl groups from histones at promoter regions chiefly correlates with gene silencing and transcriptional repression. However, previous studies have also shown gene repression $[5,12]$ as well as activation of some genes [13]. Hence, the specificity of HDACs for regulation of distinct gene programs depends on cell identity (cell state identified by gene regulation programs) and the scale of available partner proteins along with the signaling networks of the cell $[13,14]$. As an example, HDA6 in Arabidopsis, by interacting with different proteins, can regulate flowering time $[15,16]$, leaf development [17], transposon silencing [18], salt and ABA stress [19], ethylene and jasmonate signaling [20], and freezing tolerance [21]. Gene HDA19 can regulate seed maturity [22], flowering time [23], immune response, and seed dormancy by interacting with other proteins [24, 25]. HDA9 has also been reported to regulate flowering in Arabidopsis by repressing the floral activator AGL19 [26]. Additionally, HDA6 was shown to be involved in histone modifications by increasing gene expression in Arabidopsis during seed germination, salt stress, and abscisic acid treatments [27]. HDACs also showed response to various biotic stresses. In Arabidopsis, HDA19 showed induced expression when plants were challenged with $P$. syringae and the stability of induced transcripts was shown to be dependent on the levels of salicylic acid and pathogen-related 1 (NPR-1) gene expression [28].

Recent phylogenetic analysis of sequences from the HDACs superfamily RPD3/HDA1 from Arabidopsis enabled further classification into three classes, class I, class II, and class III [29]. Similarly, genome analysis of rice HDACs enabled the identification of an additional class, class IV, [30] indicating the diversity and need for further studies in other commercially important crop plants including legumes. Expression analysis of HDACs from all classes and families showed differential expression during developmental stages, environmental stresses, and hormonal stimuli [31, 32].

The long-term goal of our research is to understand epigenetic modifications in common bean during infection by the rust fungal pathogen. In this study, we report progress on our understanding of the role of HDACs during infection of common bean with the bean rust pathogen, $U$. appendiculatus race 53 . We focus on understanding and quantifying total HDAC activity present in mock inoculated (MI) and inoculated (I) leaf tissues at 0,12 , and 84 hours after inoculation (hai) and analyses of the expression profiles of selected genes from each known plant HDAC family.

\section{Materials and Methods}

2.1. Plant Materials and Pathogen Infection. The common bean cultivar "Sierra" is resistant to common bean rust race 53 (Figure $1(\mathrm{a})$ ) and carries the rust resistant genes Ur-3 and Crg [33, 34]. Sierra exhibits a hypersensitive response upon inoculation with race 53, the cultivar "Olathe," which is recessive at $U r$-3 is susceptible to this pathogen race. The genotype crg, a susceptible mutant derived from Sierra which carries a mutation at the Crg locus also develops rust like symptoms (rusty-yellow or bright orange spots) on leaves. Both Olathe and crg were used in this experiment as control for demonstrating successful inoculations.

Plants were grown in the greenhouse as per Melmaiee et al. [35]. When plants were ten days old at the primary leaf stage, half of the seedlings from each genotype were inoculated with $U$. appendiculatus race 53 spores with $1 \%$ Tween 20 on the adaxial and abaxial sides of the two leaves and another half of the seedlings were mock inoculated (MI) with only 1\% Tween 20 along with Olathe and crg as inoculation experimental controls. After inoculation, plants were placed in a growth chamber with high humidity (approximately $90 \%)$ to facilitate the establishment of fungal growth. Sierra leaf samples were collected at 0,12 , and 84 hai along with MI samples for analysis as shown in Figure 2 for nuclear extraction and total RNA isolation. The above time points were chosen based on our previous experiments [35, 36]. For each sampling time, leaves were pooled from three different plants (one leaf from each plant) and utilized for colorimetric assays, and three leaves from another set of three plants were collected and flash frozen for gene expression analyses. The entire experiment was repeated twice yielding two biological replications of the study.

2.2. Scanning Electron Microscopy. Symptomatic leaves were collected from susceptible mutant genotype crg (derived from Sierra) [33], dehydrated with ethanol, and mounted on stubs using carbon filled adhesive. The dehydrated specimens were coated with gold palladium by sputter coater 108 auto (Cressington Scientific Instruments Ltd., Watford, UK) and observed with an analytical scanning electron microscope S2600N (Hitachi High Technologies America, Inc., Schaumburg, IL) located in the College of Agriculture \& Related Sciences at DSU.

2.3. Nuclear Extraction. Nuclear extractions were carried out from MI and I leaf samples using the EpiQuik Nuclear Extraction kit 1 (Epigentek Group Inc., Farmingdale, NY). Approximately one gram of leaf samples (either flash frozen or fresh) was cut into small pieces and submerged in a $1: 10$ diluted nuclear extraction buffer 1 (NE1) with 1x dithiothreitol (DTT) in a mortar and ground thoroughly until all the leaf samples became fine paste. Samples were incubated on ice for 15 minutes and centrifuged for 10 minutes at $11,000 \times \mathrm{g}$ to obtain a nuclear pellet. The supernatant was removed and $500 \mu \mathrm{L}$ of nuclear extraction buffer 2 (NE2) containing 1x DTT was added to the nuclear pellet and incubated for another 15 minutes on ice. During this time samples were vortexed for $5 \mathrm{sec}$ at three-minute intervals to increase nuclear protein concentration. Samples were then centrifuged at $14,000 \mathrm{~g}$ for 10 minutes at $4^{\circ} \mathrm{C}$, and the nuclear protein was quantified with Qubit fluorometer (Life Technologies, Grand Island, NY) and stored at $-80^{\circ} \mathrm{C}$ for further analyses. 

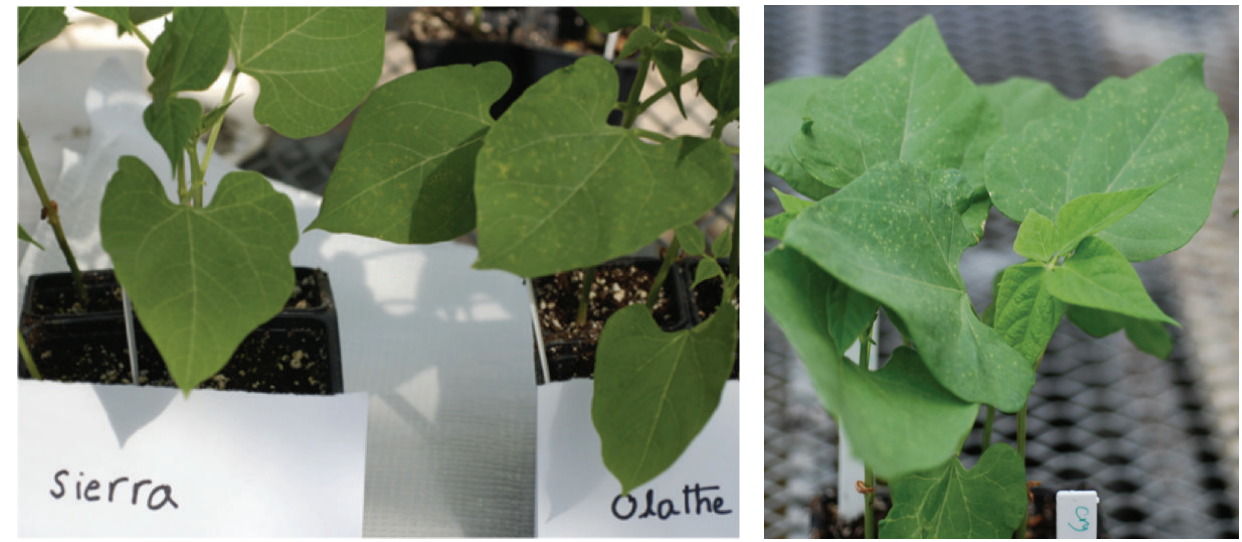

(a)

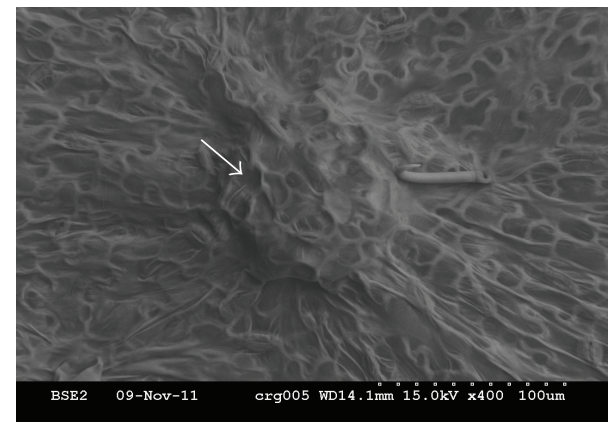

(b)

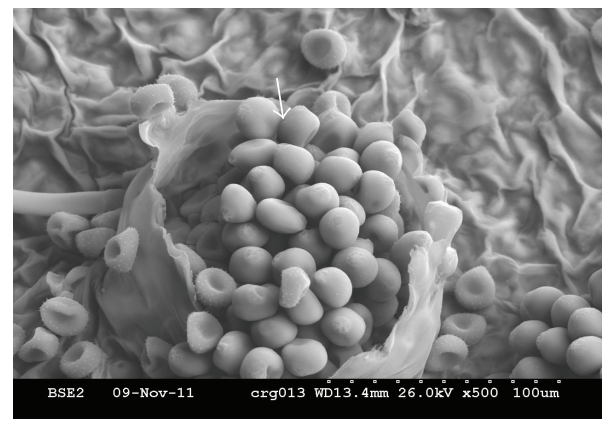

(d)

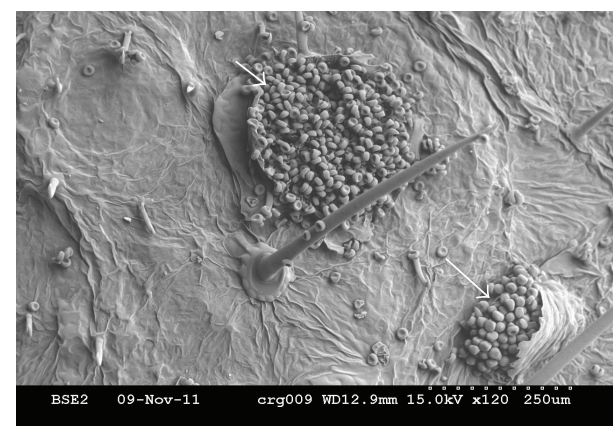

(c)

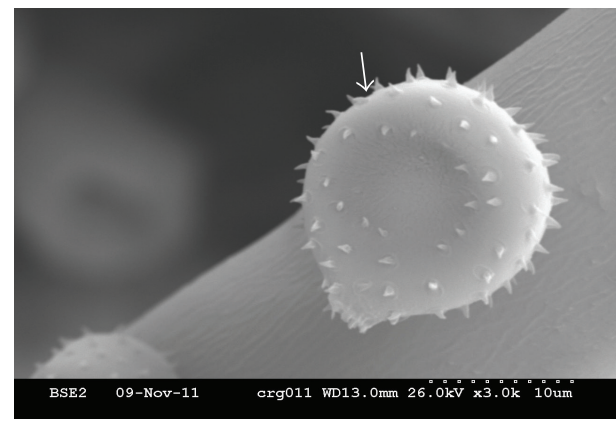

(e)

Figure 1: Common bean rust symptoms, rust pustules, and spores. (a) Ten-day-old seedlings at the primary leaf stage were inoculated with rust race 53. The susceptible genotype Olathe and susceptible mutant crg developed visible rust like symptoms after approximately 10 days of inoculation, whereas the resistant genotype Sierra was asymptomatic as expected. The genotypes Olathe and crg were used as an inoculation experimental control only and are shown here. (b-e) Scanning electron microscope pictures from a leaf of a rust susceptible genotype crg after symptoms were developed. Picture (b) is an unopened rust pustule. ((c) and (d)) Pustules which burst open at 120 and 500 times' magnification. (e) Rust spore under 3000 times' magnification. White arrows point to the pustules and fungal spores.

2.4. Quantification of HDACs by Colorimetric Method. HDAC activity was measured with $1.545 \mu \mathrm{g}$ of nuclear protein extract following the manufacturers protocol (HDAC Assay Kit, colorimetric, Active Motif, Carlsbad, CA). As suggested in the protocol for samples with potentially low HDACs, we extended the initial HDAC reaction time to three hours. Since the kit was developed based on nuclear extracts from mammalian cells, we envisioned that extending the incubation time will help complete the deacetylation reaction. Samples were measured in triplicate (standards were measured in duplicate) using $\mathrm{EPOCH}$ colorimetric plate reader (Biotek, Winooski, VT) at $405 \mathrm{~nm}$. In the assay reaction, a short peptide substrate was added along with the nuclear extract and other reagents as per the protocol. This substrate contains acetylated lysine residues and can be deacetylated by most HDAC enzymes. Active HDACs from the experimental samples would then bind to the added substrate by removing acetyl groups from the substrate. This reaction then yielded an HDAC-deacetylated colored product, which was measured by the colorimetric plate 
TABLE 1: Selected representative HDAC genes for expression analysis. GenBank protein accession number and corresponding predicted common bean homologs along with location on the common bean genome.

\begin{tabular}{|c|c|c|c|c|c|}
\hline $\begin{array}{l}\text { Gene } \\
\text { family/class }\end{array}$ & $\begin{array}{l}\text { GenBank } \\
\text { protein } \\
\text { accession } \\
\text { number }\end{array}$ & $\begin{array}{l}\text { Phytozome common bean } \\
\text { CDS number }\end{array}$ & $\begin{array}{l}\text { HDACs } \\
\text { names }\end{array}$ & $\begin{array}{l}\text { Location on the } \\
\text { bean genome } \\
\text { (chromosome) }\end{array}$ & $\begin{array}{l}\text { Corresponding } \\
\text { model organism }\end{array}$ \\
\hline \multirow[t]{2}{*}{$\begin{array}{l}\text { RPD3/HDA1 } \\
\text { family class I }\end{array}$} & $\begin{array}{l}\text { AAC } 50038 \\
\text { AAK0712.1 }\end{array}$ & Phvul.009G115300.1 & $P v R P D 3$ & 9 & $\begin{array}{l}\text { Zea mays } \\
\text { Oryza sativa }\end{array}$ \\
\hline & BAB10553 & Phvul.003G203800.1 & $P v H D A 6$ & 6 & A. thaliana \\
\hline Class II & $\begin{array}{l}\text { NP_200915 } \\
\text { NP } 200914\end{array}$ & Phvul.003G185200.1 & PvHDA18 & 3 & A. thaliana \\
\hline Class III & AAD40129 & Phvul.001G034500.2 & PvHDA2 & 1 & A. thaliana \\
\hline HD2 family & AAB70032 & Phvul.001G186300.1 & PvHD2 & $\begin{array}{c}\text { sg0.contig } \\
\text { 03923: } 2769-5777\end{array}$ & A. thaliana \\
\hline SIR2 family & BAB09243 & Phvul.006G057700.1 & PvSRT1 & 6 & A. thaliana \\
\hline
\end{tabular}

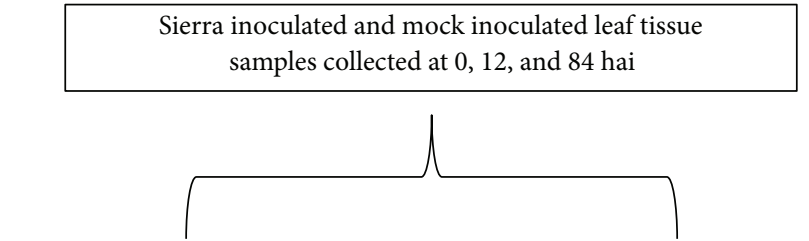

Nuclear protein extraction

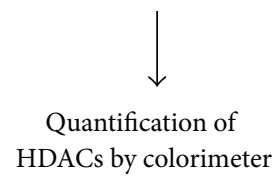

Total RNA extraction

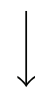

Quantitative

real-time PCR analysis

FIGURE 2: Flowchart outlining the experiment in this study. Same sets of materials from each biological replicate were utilized to perform colorimetric analysis and gene expression studies.

reader. The amount of deacetylated product in the reaction is directly proportional to the amount of active HDAC enzymes present in our samples [37].

2.5. Selection of HDAC Gene Sequences and Primer Design. Representative proteins from each HDAC family or class were selected based on the previous reports [29]. The GenBank protein accession numbers AAC50038, AAK0712.1, BAB10553, NP_200914, NP_200915, and AAD40129, from RPD3 (reduced potassium dependency)/HDA1 (histone deacetylase 1) family, AAB70032 from HD2 family, and BAB09243 from SIR2 (Silent Information Regulator 2) were selected for gene expression analysis. All of these sequences were derived from Arabidopsis thaliana except AAK01712.1 and AAC50038, which were derived from rice (Oryza sativa) and maize (Zea mays).

GenBank protein accession numbers were used to extract model organism protein sequences from NCBI database and these sequences were compared against the common bean predicted proteome derived from the common bean genomesequencing project from http://www.phytozome.org/ [38].
TABle 2: Primer sequences utilized for qRT-PCR.

\begin{tabular}{|c|c|}
\hline $\begin{array}{l}\text { Predicted common } \\
\text { bean gene }\end{array}$ & Primer sequences \\
\hline Phvul.009G115300.1 & $\begin{array}{l}\text { ACATGAGCGTGTTCTGTACGTGGA } \\
\text { TCAGCACCGCATTGGAGAACTACT }\end{array}$ \\
\hline Phvul.003G203800.1 & $\begin{array}{l}\text { CATCCGCATGGCGCACAATCTTAT } \\
\text { ACCCAACCTGTCACCAGACAATGA }\end{array}$ \\
\hline Phvul.003G185200.1 & $\begin{array}{l}\text { TCTGCGGTTAGTGCATTCCAGAGT } \\
\text { GGGTCACCAACAGCTGCATCAAAT }\end{array}$ \\
\hline Phvul.001G034500.2 & $\begin{array}{l}\text { TCGGCATAGAGAAACTGCATCCGT } \\
\text { ACCACCTGAAGTGAGCATGACGAT }\end{array}$ \\
\hline Phvul.001G186300.1 & $\begin{array}{l}\text { AACTGGTAGCCCTGAACGTGAAGT } \\
\text { TCCCATTGGCAGCACTAACTGGAA }\end{array}$ \\
\hline Phvul.006G057700.1 & $\begin{array}{l}\text { CTTGCCAGAAGCATCACTGCCATT } \\
\text { GGCAAGTTGCACGCTGGAGTTATT }\end{array}$ \\
\hline TC362 & $\begin{array}{l}\text { GCTCTCCATTTGCTCCCTGTT } \\
\text { TGAGCAATTTCAGGCACCAA }\end{array}$ \\
\hline
\end{tabular}

The best match was selected and coding sequences (CDS) were extracted for further analysis (Supplementary File 1) (see Supplementary Material available online at http://dx.doi.org/ 10.1155/2015/153243). Proteins AAK0712.1 and AAC 50038.1 matched the same common bean CDS Phvul.009G115300.1 and proteins NP_200914 and NP_200915 matched Phvul.003G185200.1 and other proteins matched different common bean sequences as shown in Table 1. For convenience, we named these CDS (referenced in this study as Phaseolus vulgaris HDACs) as mentioned in column 4 of Table 1. For gene expression analysis, primers were designed with Primer quest software as in Table 2 and tested with common bean genomic DNA (Figure 4(a)).

2.6. Total RNA Isolation and cDNA Synthesis. Total RNA was extracted using TRIzol reagent (Invitrogen, Carlsbad, CA) from flash frozen pooled leaf tissues (three leaves from three plants) as per manufacturer's protocol and the RNA was digested with the enzyme rDNAse (Life Technologies, Grand Island, NY) to remove any contaminating DNA. Absence 
of genomic DNA was confirmed with known primers that can amplify intronic regions as mentioned previously [39]. Total RNA was used to synthesize cDNA with ProtoScript M-MuLV First Strand cDNA synthesis kit (New England BioLabs, Beverly, MA).

2.7. Gene Expression Analysis by Quantitative Real-Time PCR ( $q R T-P C R)$. Concentrations of cDNA were equalized for all the samples under consideration and qRT-PCR analysis was carried out on Applied Biosystems 7500 real-time machine (Foster City, CA) using SYBR Green dye. Gene expression was normalized to the housekeeping gene ubiquitinconjugating enzyme E2 UBC9 (TC362) [40] and included in each PCR run. The whole experiment was replicated twice with three technical replications for each sample analyzed. Gene expression analysis was carried out by comparative $2^{-\Delta \Delta C T}$ method [41] and used to calculate expression values and indicated in fold changes. Student's $t$-test was performed with a $P$ value cutoff of 0.05 .

\section{Results and Discussion}

3.1. HDACs Activity during Fungal Infection. Activity of total HDAC enzymes was quantified by a colorimetry-based assay. We collected leaf tissue from inoculated and mock inoculated rust resistant genotype Sierra (tissue pooled from 3 leaves for each time point) at 0,12 , and 84 hai, from which nuclear extracts were then isolated and processed. A standard curve was generated using the standards provided with the HDAC Activity Kit and optical density (OD) values of the samples from MI plants and I plants were then extrapolated. The mean values from two independent biological replicates were calculated (Figure 3). Colorimetric analysis revealed that there is a reduction in the amount of active HDACs (37.68 nmol) in the inoculated samples at 12 hai compared to mock inoculated plants $(48.97 \mathrm{nmol})$, whereas at 84 hai the activity was approximately $37.0 \mathrm{nmol}$ in both the samples.

The reduction in overall HDAC activity at 12 hai suggests that there may be less deacetylation reactions at this time point and more uncoiled DNA was available for transcription as there will be a demand for induction of stress resistant genes at this time. However, colorimetric analysis indicates that HDACs activity changes throughout the course of rust infection in common bean plants and differs between plants challenged or not challenged with the fungal pathogen.

3.2. Identification of Common Bean Homologous Sequences. HDAC protein sequences were obtained from Arabidopsis and other plant species from GenBank using corresponding protein accession numbers. These protein sequences were searched against the common bean predicted protein database and common bean CDS were obtained (Supplementary File 1) for gene expression analysis. Since common bean CDS were derived by bioinformatics analysis, corresponding primers were initially amplified with genomic DNA of the common bean (Figure 4(a)) and then with cDNA derived from the experimental samples (Figure 4(b)), which were

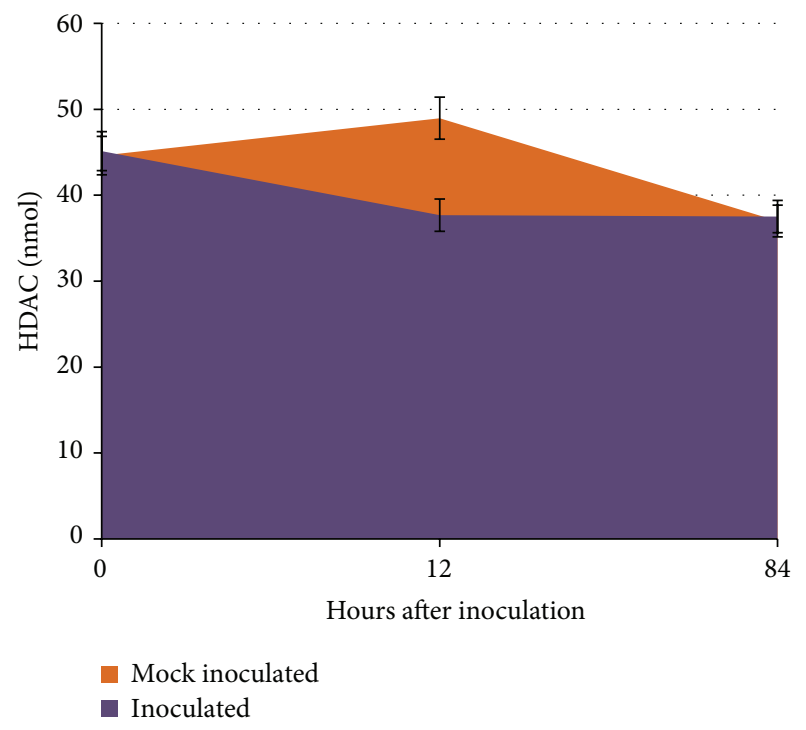

FIGURE 3: HDAC activity between mock inoculated and inoculated common bean. Total HDAC activity was measured based on the optical density (OD) and the amount of activity was determined based on the standard curve. The experimental values differ significantly with a probability value of $0.05 \%$. The error bars represent standard deviation.

used for qRT-PCR analysis. All the genes tested were amplified in both the genomic DNA and cDNA samples.

3.3. Gene Expression Analysis. Between the two genes that were studied in class 1 (RPD3 family), gene PvHDA6 showed increased expression at both 12 and 84 hai in the inoculated samples (Figure 5(a)). In the MI samples, the PvHDA6 expression was seen to be slightly increased at 12 hai and was neutral at 84 hai samples (Figure 5(b)). PvRPD3 expression was seen to be slightly increased at 12 hai in the I samples. However, both the genes showed slight reduction in expression at 84 hai in the MI samples. Gene PvHDA18 from class II HDACs showed increased expression at 12 hai and reduced expression at 84 hai in MI and I samples (Figures 5(c) and 5(d)). Class III gene PvHDA2 showed increased expression at both 12 and 84 hai in I samples whereas its expression was neutral in MI samples at both the time points (Figures 5(e) and 5(f)).

Similar results as observed in this study were seen in a Pseudomonas syringae resistant Arabidopsis plant with RPD3/HDA class gene HDA19. Increased expression levels of HDA19 were seen when plants were inoculated with the bacterial pathogen pstDC3000, a virulent strain of $P$. syringae pv. tomato [28]. HDA19 by interacting with the transcription factors WRKY38 and WRKY62 was suggested to help finetune basal defense responses to pathogen attack in Arabidopsis [28]. Contrastingly, Choi et al. [42] showed that HDA19 played a negative role in basal defense response mediated by salicylic acid-dependent signaling pathway, where they have observed increased expression of pathogen defense genes in HDA19 mutant plants.

In our analysis, gene $P v H D 2$ was neutral in its expression at 12 hai and showed decreased expression at 84 hai in both 


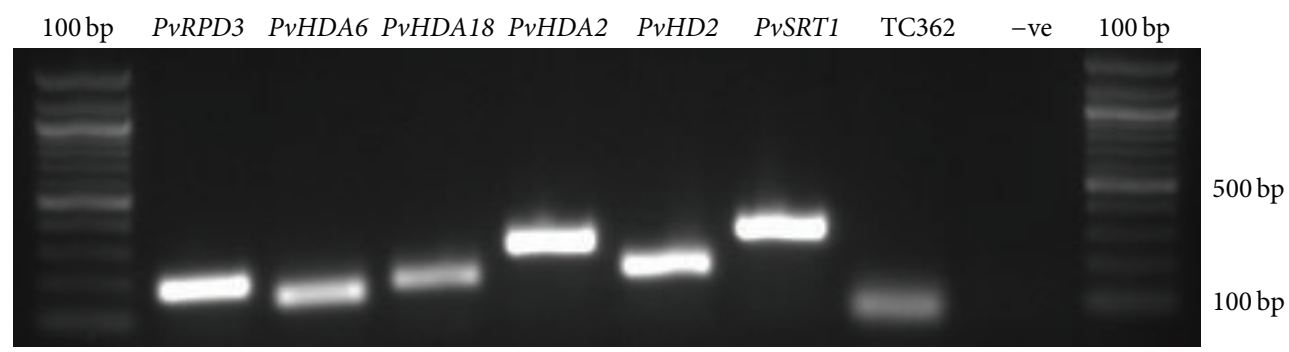

(a)
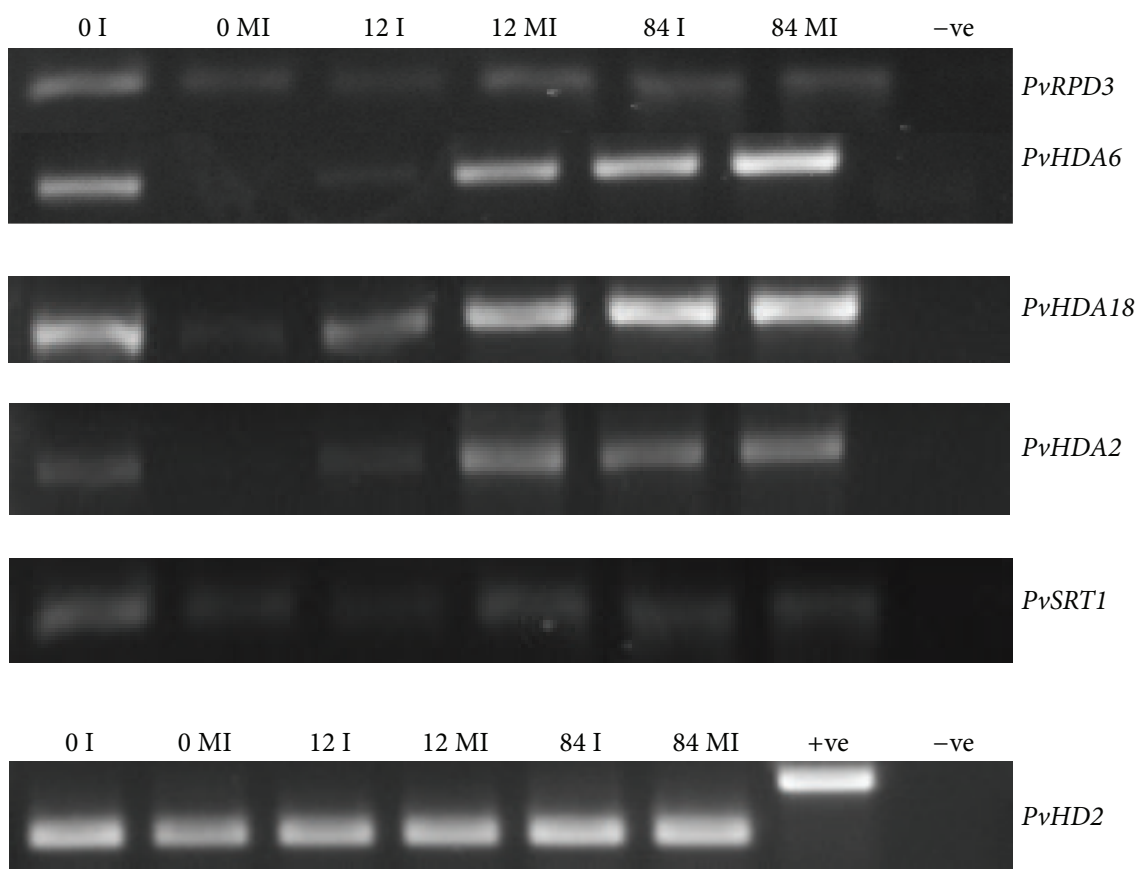

(b)

FIgURE 4: PCR amplification of HDAC genes under study. (a) Primers for the selected genes were amplified by PCR with Sierra genomic DNA and electrophoresed on a $2 \%$ agarose gel. In positive (+ve) control, reference gene TC362 primers were used and in negative (-ve) control no primers were added to the PCR reaction. (b) The same HDACs primers were tested with experimental cDNA by PCR amplification and electrophoresed on a $2 \%$ agarose gel. In +ve control, genomic DNA was used instead of cDNA and in -ve control no primers were added to the PCR reaction.

MI and I leaf samples (Figures $5(\mathrm{~g})$ and $5(\mathrm{~h})$ ). In a recent report, the tobacco $N t H D 2 a$ and $N t H D 2 b$ genes showed a rapid and strong reduction in their expression after treating the tobacco cells with cryptogein, an elicitor of tobacco defense and cell death [43].

Based on earlier findings, the HD2 class is plant specific and found only in plants [29, 44]. Differential expression of the barley HD2 genes (HvHDAC2 and HvHDAC2-2) was observed in different tissues and during seed development in barley [32] and they also exhibited differential expression in barley cultivars with varying seed size. In the same study, these genes responded to plant stress hormones such as jasmonic acid (JA), abscisic acid (ABA), and salicylic acid (SA) suggesting a possible role in epigenetic regulation due to biotic and abiotic stresses and during seed development.

Gene PvSRT1 from the SIR2 family showed contrasting expression at 12 hai, for which the expression levels were decreased in I samples and increased in MI samples. However we observed that the expression levels were increased at 84 hai in both the samples as in Figures 5(i) and 5(j). SIRT1 was reported to regulate miRNA in Alzheimer's disease patients $[45,46]$. SIR2 genes were found to be highly expressed in highly proliferating stages such as the seedling and developing panicle stages [47]. In the current study, we have used 10-day-old common bean seedlings for inoculation; hence this might be a possible reason for the presence of a higher quantity of SIRT proteins overall. This may be why the trends of active HDACs and the expression profiles of SIRT gene are similar. Additionally, we note that in this study we were able to measure one representative gene ( $P v S R T 1)$ from this class, and it will be interesting to measure the second gene. Pandey et al. [29] pointed out that only two genes from the SIRT class are currently known in plants in this class. Another consideration for future quantification experiments would be to determine protein turnover changes. 


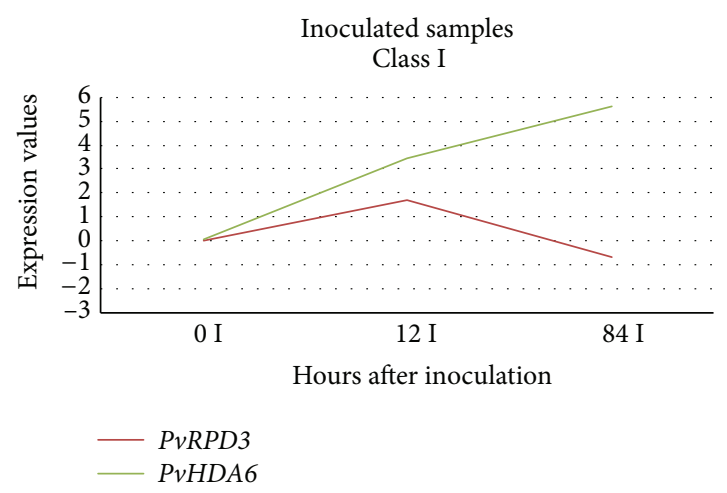

(a)

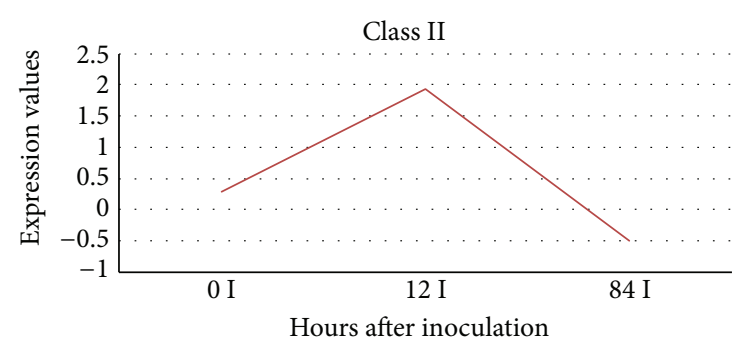

(c)

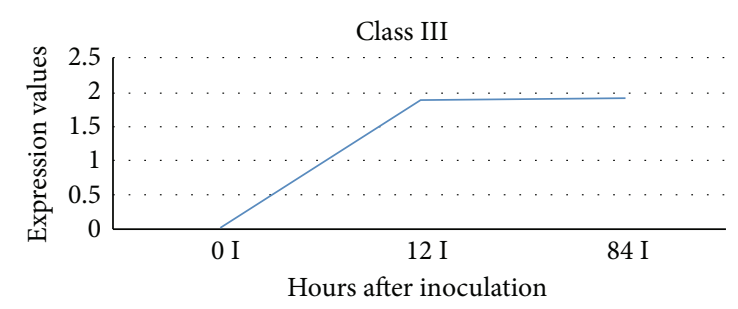

- PvHDA2

(e)

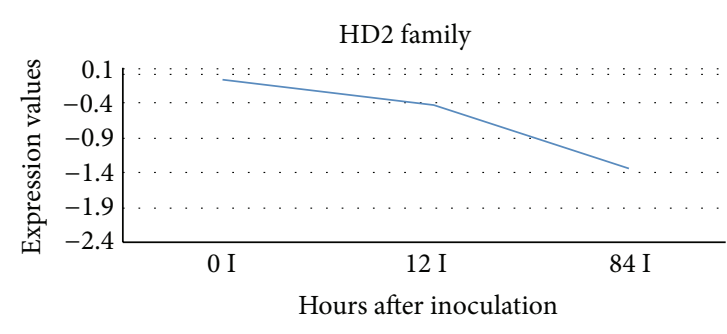

- PvHDA2

(g)

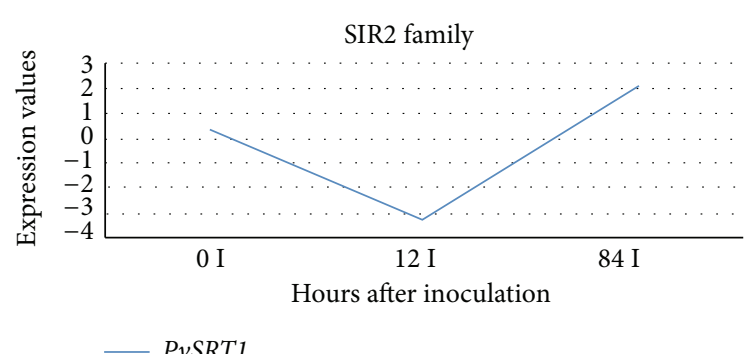

(i)

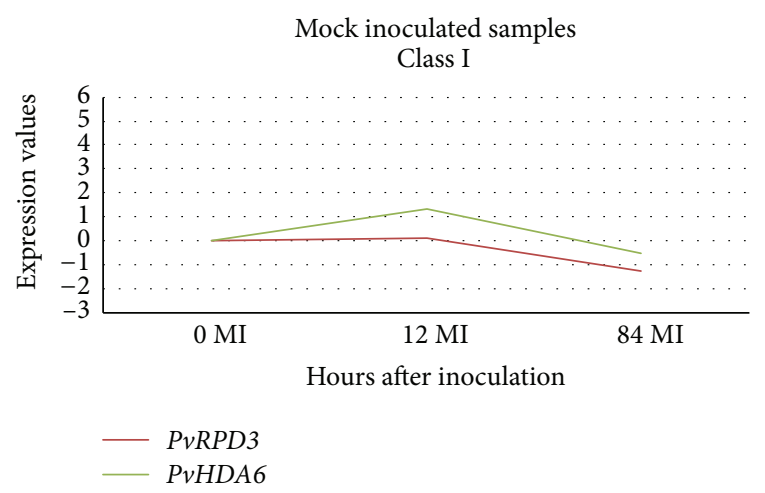

(b)

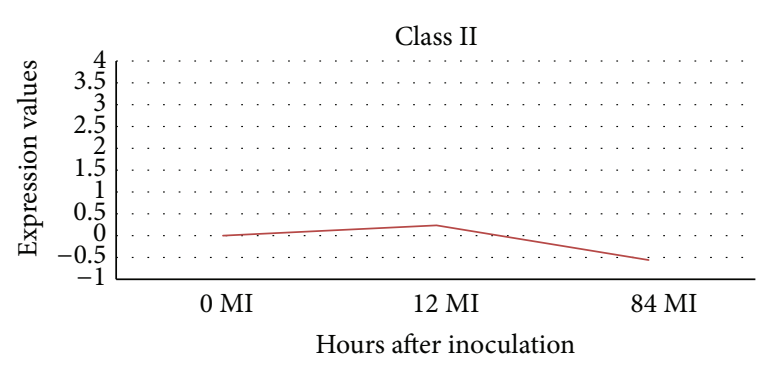

(d)

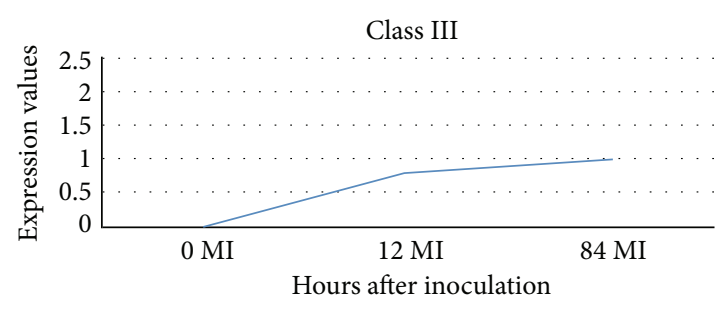

- PvHDA2

(f)

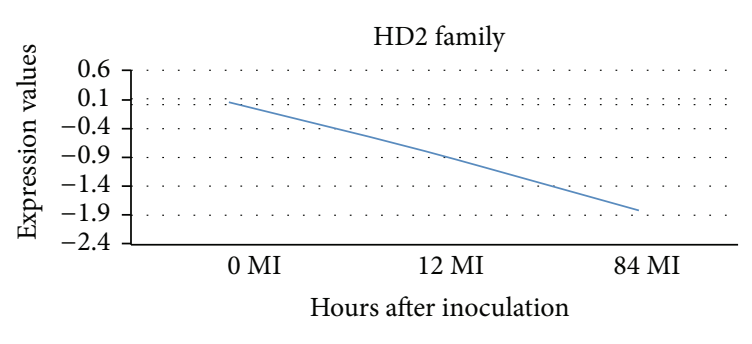

(h)

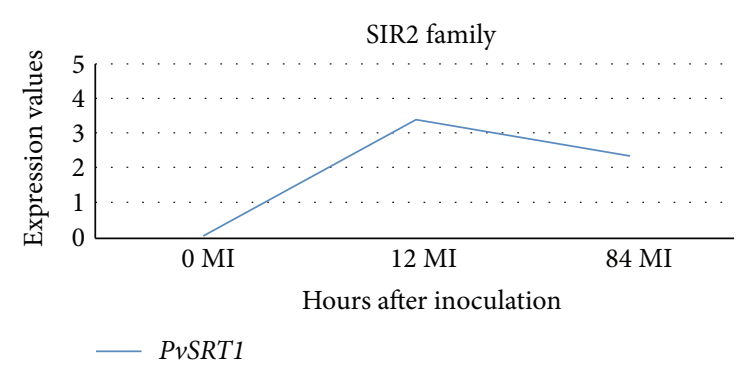

(j)

FIGURE 5: qRT-PCR analysis of HDAC genes. Figures on the left-hand side are from inoculated samples, while figures on the right-hand side are from mock inoculated samples. Sampling time points are shown in $x$-axis and $\Delta \Delta C T$ values are shown in $y$-axis. Sierra 0 hai mock inoculated samples and the endogenous gene TC362 were used for calculating expression values. 
Reduced expression of the rice SIR2 family gene OsSRT1 by specific RNA interference increased histone H3K9 acetylation, decreased $\mathrm{H} 3 \mathrm{~K} 9$ dimethylation, and also led to the development of cell death and symptoms related to plant hypersensitive response during incompatible interaction with pathogen [47]. Interestingly, in our study, PvSIRT1 showed decreased gene expression at 12 hai in leaves of inoculated plants in the bean genotype that also exhibits hypersensitive response.

HDACs play an important role in plant growth, development [48], flowering, seed maturity, and defense/tolerance to biotic and abiotic stresses. Each HDAC gene has unique functions and these genes substitute or complement each other's function. A recent observation indicated that rice HDAC genes showed more divergent functions than their homologs in Arabidopsis [49] and the same study also showed that their expression is tissue/organ specific in rice.

Based on the available literature, HDAC genes interact with histone and nonhistone proteins as well as other regulatory elements. HDA6 has been reported to interact with small interfering RNAs (siRNAs) that are generated through the RdDM pathway to suppress gene activity [50, 51]. HDA9 has been reported to regulate flowering in Arabidopsis by repressing flower-activating gene AGL 19 [26]. Histone deacetylase HDA6 is required for freezing tolerance [7]. HDA19 by interacting with WRKY 38 and WRKY62 showed enhanced basal resistance to bacterial pathogen [28].

In conclusion, reduced total HDACs activity was observed at 12 hai in rust inoculated bean plants compared to mock inoculated plants. Majority of the RPD3/HDA1 family of HDACs studied showed increased expression at least in one time point observed after inoculation. The PvHD2 gene of plant specific HDACs did not show differential expression with inoculation and may possibly be developmentally regulated. Additionally, the PvSIRT1 gene showed reduced expression at 12 hai in inoculated samples. Epigenetic analysis in common bean itself is in its infancy. This is one of the first attempts to try to understand HDACs gene regulation in common bean. As HDACs play important roles in chromatin modification, in normal plant developmental process, and in biotic/abiotic responses, our findings can be helpful to study other commercially important legume crops.

\section{Disclosure}

The funding agencies USDA or NSF were not involved in designing and implementing the experiments.

\section{Conflict of Interests}

The authors declare that there is no conflict of interests regarding the publication of this paper.

\section{Acknowledgments}

The authors acknowledge USDA funding through Grant nos. 2007-38814-18458 and 2008-38814-04735 to Venu (Kal) Kalavacharla. They also acknowledge National Science Foundation Grant no. DBI-1003917 and Delaware EPSCoR Grant nos. IIA-1301765 and EPS-0814251 and the State of Delaware to Venu (Kal) Kalavacharla. Additionally, they acknowledge members of the Molecular Genetics \& Epigenomics Laboratory (MGEL) at DSU and the College of Agriculture \& Related Sciences for support of this research activity.

\section{References}

[1] M. Paris, M. Porcelloni, M. Binaschi, and D. Fattori, "Histone deacetylase inhibitors: from bench to clinic," Journal of Medicinal Chemistry, vol. 51, no. 6, pp. 1505-1529, 2008.

[2] P. A. Marks, "Discovery and development of SAHA as an anticancer agent," Oncogene, vol. 26, no. 9, pp. 1351-1356, 2007.

[3] O. Pontes, R. J. Lawrence, M. Silva et al., "Postembryonic establishment of megabase-scale gene silencing in nucleolar dominance," PLoS ONE, vol. 2, no. 11, Article ID el157, 2007.

[4] L. M. Smith, O. Pontes, I. Searle et al., "An SNF2 protein associated with nuclear RNA silencing and the spread of a silencing signal between cells in Arabidopsis," The Plant Cell, vol. 19, no. 5, pp. 1507-1521, 2007.

[5] X. Liu, S. Yang, M. Zhao et al., "Transcriptional repression by histone deacetylases in plants," Molecular Plant, vol. 7, no. 5, pp. 764-772, 2014.

[6] R. L. Momparler, "Cancer epigenetics," Oncogene, vol. 22, no. 43, pp. 6479-6483, 2003.

[7] J.-M. Kim, T. K. To, and M. Seki, "An epigenetic integrator: new insights into genome regulation, environmental stress responses and developmental controls by Histone Deacetylase 6," Plant and Cell Physiology, vol. 53, no. 5, pp. 794-800, 2012.

[8] P. L. Jones, G. J. C. Veenstra, P. A. Wade et al., "Methylated DNA and $\mathrm{MeCP} 2$ recruit histone deacetylase to repress transcription," Nature Genetics, vol. 19, no. 2, pp. 187-191, 1998.

[9] S. H. Rangwala and E. J. Richards, "Differential epigenetic regulation within an Arabidopsis retroposon family," Genetics, vol. 176, no. 1, pp. 151-160, 2007.

[10] B. P. May, Z. B. Lippman, Y. Fang, D. L. Spector, and R. A. Martienssen, "Differential regulation of strand-specific transcripts from Arabidopsis centromeric satellite repeats," PLoS Genetics, vol. 1, no. 6, article e79, 2005.

[11] P. A. Wade, A. Gegonne, P. L. Jones, E. Ballestar, F. Aubry, and A. P. Wolffe, "Mi-2 complex couples DNA methylation to chromatin remodelling and histone deacetylation," Nature Genetics, vol. 23, no. 1, pp. 62-66, 1999.

[12] D. Jiang, W. Yang, Y. He, and R. M. Amasino, “Arabidopsis relatives of the human lysine-specific Demethylasel repress the expression of FWA and Flowering Locus $C$ and thus promote the floral transition," The Plant Cell, vol. 19, no. 10, pp. 2975-2987, 2007.

[13] M. Haberland, R. L. Montgomery, and E. N. Olson, "The many roles of histone deacetylases in development and physiology: implications for disease and therapy," Nature Reviews Genetics, vol. 10, no. 1, pp. 32-42, 2009.

[14] D. Hnisz, A. F. Bardet, C. J. Nobile et al., "A histone deacetylase adjusts transcription kinetics at coding sequences during Candida albicans morphogenesis," PLoS Genetics, vol. 8, no. 12, Article ID e1003118, 2012.

[15] J.-H. Jung, J.-H. Park, S. Lee et al., “The cold signaling attenuator HIGH EXPRESSION OF OSMOTICALLY RESPONSIVE GENE1 activates FLOWERING LOCUS C transcription via chromatin remodeling under short-term cold stress in Arabidopsis," The Plant Cell, vol. 25, no. 11, pp. 4378-4390, 2013. 
[16] J. Yun, Y.-S. Kim, J.-H. Jung, P. J. Seo, and C.-M. Park, “The AT-hook motif-containing protein AHL22 regulates flowering initiation by modifying Flowering Locus T chromatin in Arabidopsis," Journal of Biological Chemistry, vol. 287, no. 19, pp. 15307-15316, 2012.

[17] M. Luo, X. Liu, P. Singh et al., "Chromatin modifications and remodeling in plant abiotic stress responses," Biochimica et Biophysica Acta (BBA)—Gene Regulatory Mechanisms, vol. 1819, no. 2, pp. 129-136, 2012.

[18] X. Liu, C.-W. Yu, J. Duan et al., "HDA6 Directly interacts with DNA methyltransferase MET1 and maintains transposable element silencing in Arabidopsis," Plant Physiology, vol. 158, no. 1, pp. 119-129, 2012.

[19] M. Luo, Y.-Y. Wang, X. Liu et al., "HD2C interacts with HDA6 and is involved in ABA and salt stress response in Arabidopsis," Journal of Experimental Botany, vol. 63, no. 8, Article ID ers059, pp. 3297-3306, 2012.

[20] Z. Zhu, F. An, Y. Feng et al., "Derepression of ethylenestabilized transcription factors (EIN3/EIL1) mediates jasmonate and ethylene signaling synergy in Arabidopsis," Proceedings of the National Academy of Sciences of the United States of America, vol. 108, no. 30, pp. 12539-12544, 2011.

[21] T. K. To, K. Nakaminami, J.-M. Kim et al., "Arabidopsis HDA6 is required for freezing tolerance," Biochemical and Biophysical Research Communications, vol. 406, no. 3, pp. 414-419, 2011.

[22] Y. Zhou, B. Tan, M. Luo et al., "HISTONE DEACETYLASE 19 interacts with $\mathrm{HSL}_{1}$ and participates in the repression of seed maturation genes in Arabidopsis seedlings," The Plant Cell, vol. 25, no. 1, pp. 134-148, 2013.

[23] X. Gu, Y. Wang, and Y. He, "Photoperiodic regulation of flowering time through periodic histone deacetylation of the florigen gene FT,” PLoS Biology, vol. 11, no. 9, Article ID e1001649, 2013.

[24] Z. Wang, H. Cao, Y. Sun et al., “Arabidopsis paired amphipathic helix proteins SNL1 and SNL2 redundantly regulate primary seed dormancy via abscisic acid-ethylene antagonism mediated by histone deacetylation," The Plant Cell, vol. 25, no. 1, pp. 149166, 2013.

[25] Z. Zhu, F. Xu, Y. Zhang et al., "Arabidopsis resistance protein $\mathrm{SNC1}$ activates immune responses through association with a transcriptional corepressor," Proceedings of the National Academy of Sciences of the United States of America, vol. 107, no. 31, pp. 13960-13965, 2010.

[26] W. Kim, D. Latrasse, C. Servet, and D.-X. Zhou, "Arabidopsis histone deacetylase HDA9 regulates flowering time through repression of AGL19," Biochemical and Biophysical Research Communications, vol. 432, no. 2, pp. 394-398, 2013.

[27] L.-T. Chen, M. Luo, Y.-Y. Wang, and K. Wu, "Involvement of Arabidopsis histone deacetylase HDA6 in ABA and salt stress response," Journal of Experimental Botany, vol. 61, no. 12, pp. 3345-3353, 2010.

[28] K.-C. Kim, Z. Lai, B. Fan, and Z. Chen, “Arabidopsis WRKY38 and WRKY62 transcription factors interact with histone deacetylase 19 in basal defense," The Plant Cell, vol. 20, no. 9, pp. 2357-2371, 2008.

[29] R. Pandey, A. Müller, C. A. Napoli et al., "Analysis of histone acetyltransferase and histone deacetylase families of Arabidopsis thaliana suggests functional diversification of chromatin modification among multicellular eukaryotes," Nucleic Acids Research, vol. 30, no. 23, pp. 5036-5055, 2002.

[30] W. Fu, K. Wu, and J. Duan, "Sequence and expression analysis of histone deacetylases in rice," Biochemical and Biophysical Research Communications, vol. 356, no. 4, pp. 843-850, 2007.
[31] K. Demetriou, A. Kapazoglou, K. Bladenopoulos, and A. S. Tsaftaris, "Epigenetic chromatin modifiers in barley: II. Characterization and expression analysis of the HDA1 family of barley histone deacetylases during development and in response to jasmonic acid," Plant Molecular Biology Reporter, vol. 28, no. 1, pp. 9-21, 2010.

[32] K. Demetriou, A. Kapazoglou, A. Tondelli et al., "Epigenetic chromatin modifiers in barley. I. Cloning, mapping and expression analysis of the plant specific HD2 family of histone deacetylases from barley, during seed development and after hormonal treatment," Physiologia Plantarum, vol. 136, no. 3, pp. 358-368, 2009.

[33] V. Kalavacharla, J. R. Stavely, J. R. Myers, and P. E. McClean, " $\mathrm{Crg}$, a gene required for Ur-3-mediated rust resistance in common bean, maps to a resistance gene analog cluster," Molecular Plant-Microbe Interactions, vol. 13, no. 11, pp. 1237$1242,2000$.

[34] K. Melmaiee, A. Todd, P. McClean et al., "Identification of molecular markers associated with the deleted region in common bean (Phaseolus vulgaris) ur-3 mutants," Australian Journal of Crop Science, vol. 7, no. 3, pp. 354-360, 2013.

[35] K. Melmaiee, A. Brown, N. Kendall, and V. Kalavacharla, "Expression profiling of wrky transcription factors in common bean during rust fungal infection," Report of the Bean Improvement Cooperative 55, 2012.

[36] V. Ayyappan, V. Kalavacharla, J. Thimmapuram et al., "Genome-wide profiling of histone modifications $\left(\mathrm{H} 3 \mathrm{~K}_{\text {me2 }}\right.$ and $\mathrm{H} 4 \mathrm{~K} 12_{\mathrm{ac}}$ ) and gene expression in rust (Uromyces appendiculatus) inoculated common bean (Phaseolus vulgaris L.)," PLoS ONE, vol. 10, no. 7, Article ID e0132176, 2015.

[37] X.-J. Yang and S. Grégoire, "Class II histone deacetylases: from sequence to function, regulation, and clinical implication," Molecular and Cellular Biology, vol. 25, no. 8, pp. 2873-2884, 2005.

[38] J. Schmutz, P. E. McClean, S. Mamidi et al., "A reference genome for common bean and genome-wide analysis of dual domestications," Nature Genetics, vol. 46, no. 7, pp. 707-713, 2014.

[39] V. Kalavacharla, Z. Liu, B. C. Meyers, J. Thimmapuram, and K. Melmaiee, "Identification and analysis of common bean (Phaseolus vulgaris L.) transcriptomes by massively parallel pyrosequencing," BMC Plant Biology, vol. 11, article 135, 2011.

[40] G. Hernández, M. Ramírez, O. Valdés-López et al., "Phosphorus stress in common bean: root transcript and metabolic responses," Plant Physiology, vol. 144, no. 2, pp. 752-767, 2007.

[41] K. J. Livak and T. D. Schmittgen, "Analysis of relative gene expression data using real-time quantitative PCR and the $2^{-\triangle \Delta C_{\mathrm{T}}}$ method," Methods, vol. 25, no. 4, pp. 402-408, 2001.

[42] S.-M. Choi, H.-R. Song, S.-K. Han et al., "HDA19 is required for the repression of salicylic acid biosynthesis and salicylic acidmediated defense responses in Arabidopsis," The Plant Journal, vol. 71, no. 1, pp. 135-146, 2012.

[43] S. Bourque, A. Dutartre, V. Hammoudi et al., “Type-2 histone deacetylases as new regulators of elicitor-induced cell death in plants," New Phytologist, vol. 192, no. 1, pp. 127-139, 2011.

[44] A. Lusser, G. Brosch, A. Loidl, H. Haas, and P. Loidl, "Identification of maize histone deacetylase HD2 as an acidic nucleolar phosphoprotein," Science, vol. 277, no. 5322, pp. 88-91, 1997.

[45] A. Zovoilis, H. Y. Agbemenyah, R. C. Agis-Balboa et al., "microRNA-34c is a novel target to treat dementias," The EMBO Journal, vol. 30, no. 20, pp. 4299-4308, 2011. 
[46] N. Schonrock, Y. D. Ke, D. Humphreys et al., "Neuronal microrna deregulation in response to Alzheimer's disease amyloid- $\beta$," PLoS ONE, vol. 5, no. 6, Article ID el1070, 2010.

[47] L. Huang, Q. Sun, F. Qin, C. Li, Y. Zhao, and D.-X. Zhou, "Down-regulation of a Silent Information Regulator2-related histone deacetylase gene, OsSRT1, induces DNA fragmentation and cell death in rice," Plant Physiology, vol. 144, no. 3, pp. 15081519, 2007.

[48] H. H. Tai, G. C. C. Tai, and T. Beardmore, "Dynamic histone acetylation of late embryonic genes during seed germination," Plant Molecular Biology, vol. 59, no. 6, pp. 909-925, 2005.

[49] Y. Hu, F. Qin, L. Huang et al., "Rice histone deacetylase genes display specific expression patterns and developmental functions," Biochemical and Biophysical Research Communications, vol. 388, no. 2, pp. 266-271, 2009.

[50] W. Aufsatz, M. F. Mette, J. van der Winden, M. Matzke, and A. J. M. Matzke, "HDA6, a putative histone deacetylase needed to enhance DNA methylation induced by double-stranded RNA," The EMBO Journal, vol. 21, no. 24, pp. 6832-6841, 2002.

[51] W. Aufsatz, T. Stoiber, B. Rakic, and K. Naumann, "Arabidopsis histone deacetylase 6: a green link to RNA silencing," Oncogene, vol. 26, no. 37, pp. 5477-5488, 2007. 

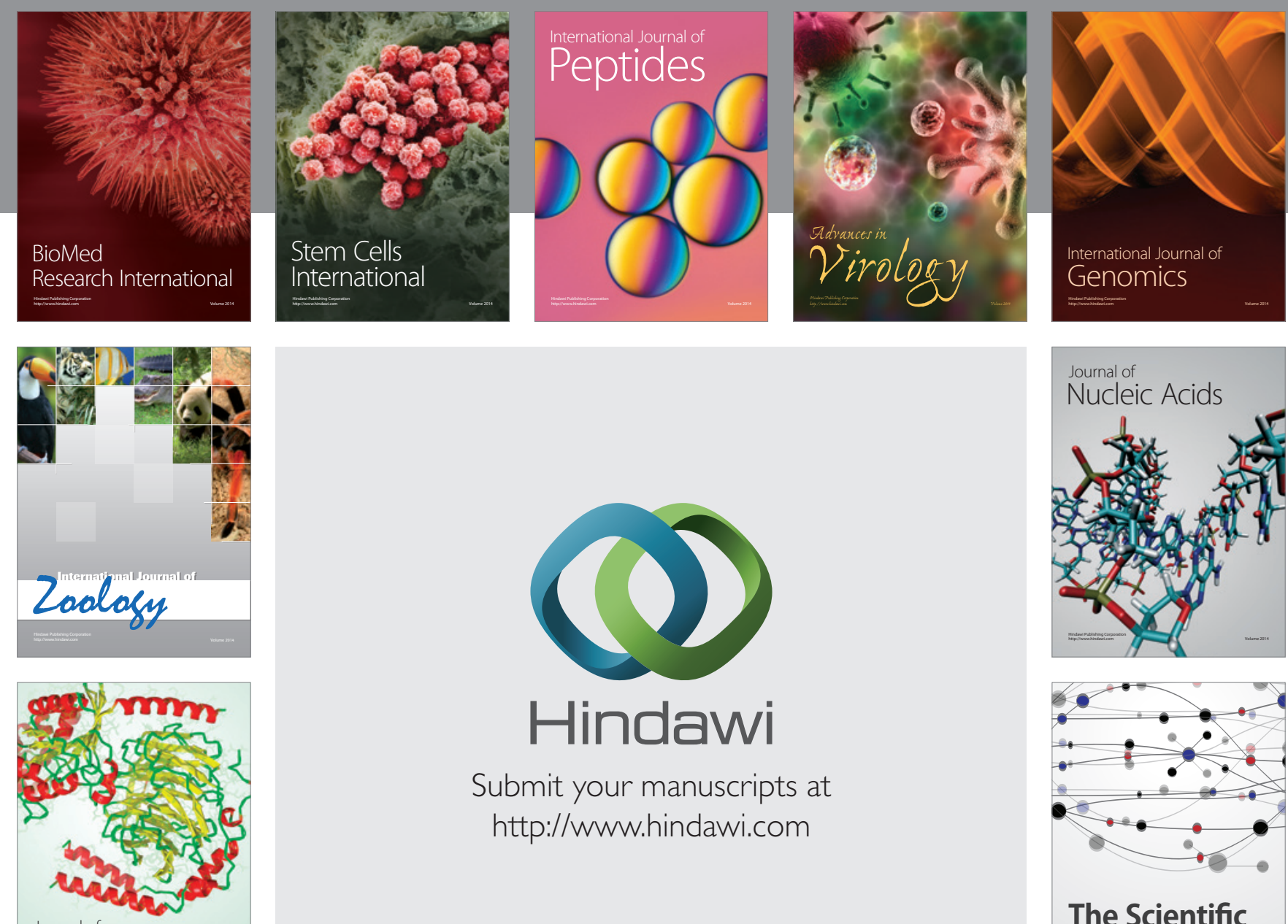

Submit your manuscripts at

http://www.hindawi.com

Journal of
Signal Transduction
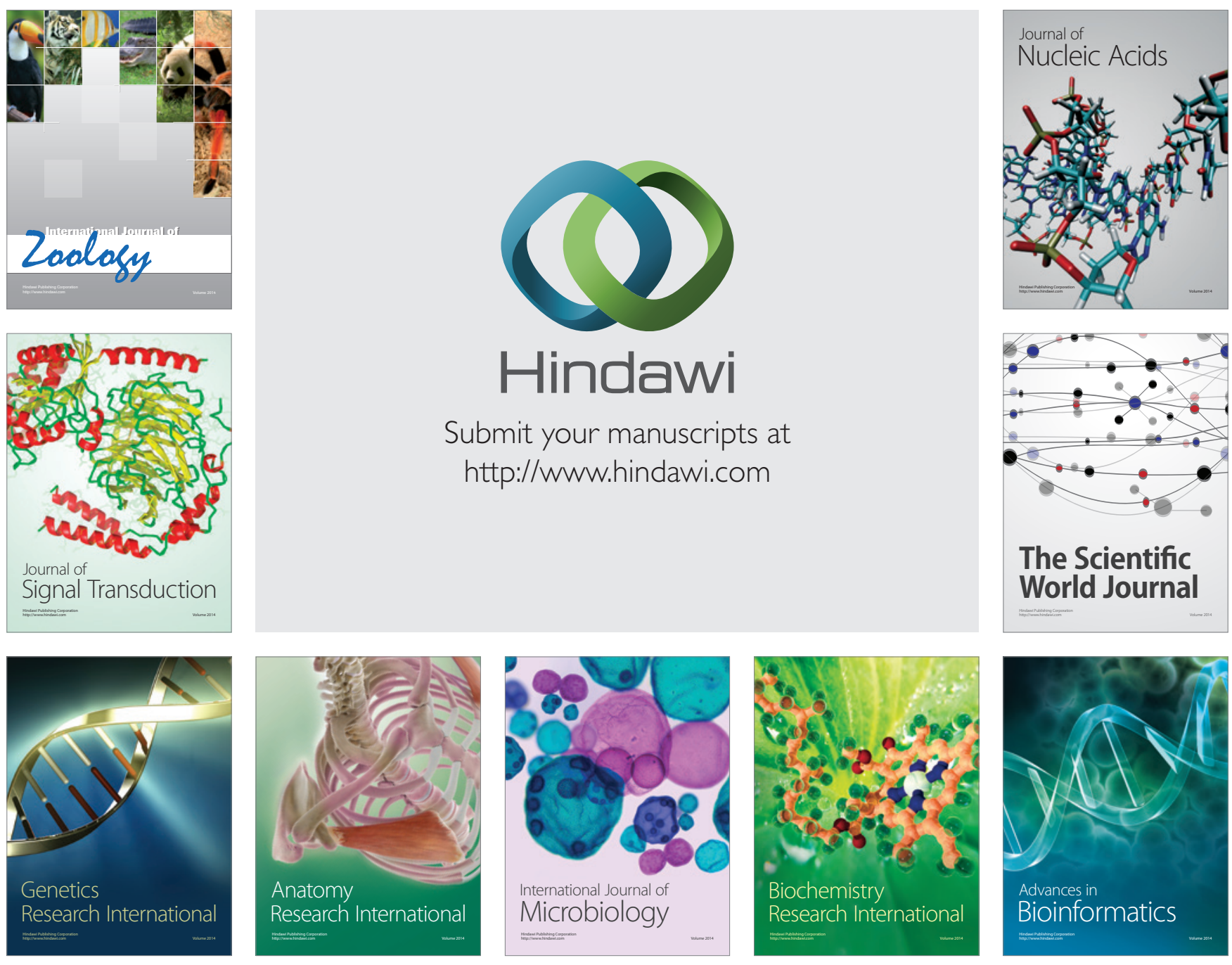

The Scientific World Journal
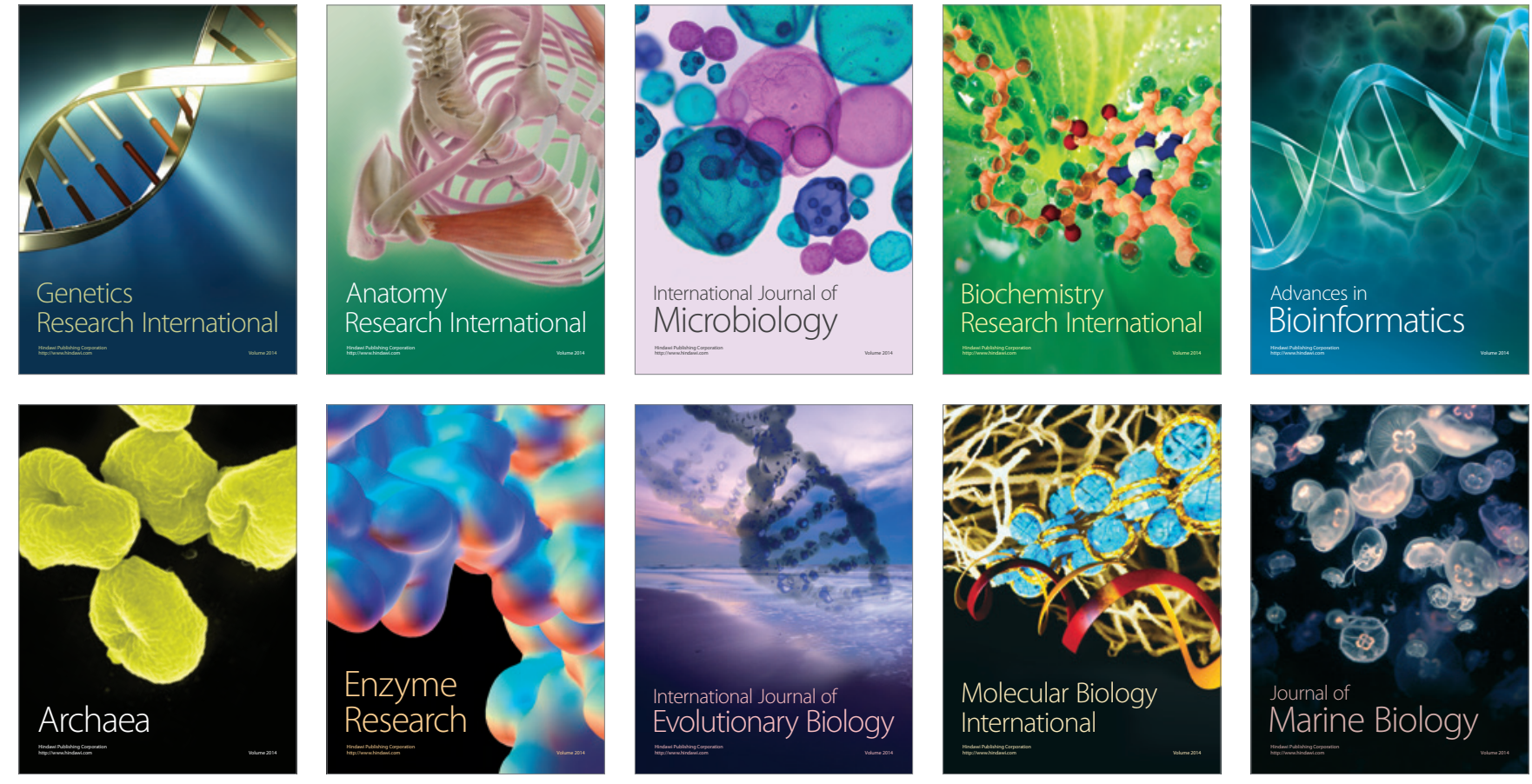